\title{
A música como instrumento de controle da ansiedade no idoso em instituições de
}

\section{longa permanência}

\author{
Music as an instrument to control anxiety in the elderly in long-stay institutions \\ La música como instrumento para el control de la ansiedad en ancianos en instituciones de larga \\ estancia
}

Recebido: 03/06/2021 | Revisado: 13/06/2021 | Aceito: 16/06/2021 | Publicado: 02/07/2021

Janaina Oliveira Morais

ORCID: https://orcid.org/0000-0002-4315-2691 Centro Universitário de Ciências e Tecnologia do Maranhão, Brasil E-mail: oliveira.janana@ hotmail.com

Kaio Germano Sousa da Silva

ORCID: https://orcid.org/0000-0003-4236-6230

Centro Universitário de Ciências e Tecnologia do Maranhão, Brasil

E-mail: kaiogsds@hotmail.com

Ana Cristina Jácome Castelo Gomes

ORCID: https://orcid.org/0000-0002-3098-2872

Centro Universitário de Ciências e Tecnologia do Maranhão, Brasil

E-mail: anajacomebb4@gmail.com

Michelly de Sousa Ferreira

ORCID: https://orcid.org/0000-0001-5386-3377

Centro Universitário de Ciências e Tecnologia do Maranhão, Brasil

E-mail: michellysousacx@hotmail.com

Auricelia Fernandes Silva Morais

ORCID: https://orcid.org/0000-0002-3281-4944

Centro Universitário de Ciências e Tecnologia do Maranhão, Brasil

E-mail: fernandesmorais2009@hotmail.com

Gercineide Ferreira Soares

ORCID: https://orcid.org/0000-0001-5986-6536

Centro Universitário de Ciências e Tecnologia do Maranhão, Brasil

E-mail: jessybizlive@gmail.com

Anastácia Tavares da Silva

ORCID: https://orcid.org/0000-0001-5106-4926

Centro Universitário de Ciências e Tecnologia do Maranhão, Brasil

E-mail: anastaciapedrolulu@gmail.com

Eduardo Brito da Silva

ORCID: https://orcid.org/0000-0002-8571-7806

Centro Universitário de Ciências e Tecnologia do Maranhão, Brasil

E-mail: eduzinhobds@gmail.com

Cristina Soares Oliveira

ORCID: https://orcid.org/0000-0002-4917-6811

Centro Universitário de Ciências e Tecnologia do Maranhão, Brasil

E-mail: csoaresoliveira27@gmail.com

Sabrina de Paula Alves de Morais

ORCID: https://orcid.org/0000-0001-6751-635X

Universidade Federal do Piauí, Brasil

E-mail: saabrina_morais@hotmail.com

Pedro Vitor Mendes Santos

ORCID: https://orcid.org/0000-0002-2249-1440

Universidade Estadual do Maranhão, Brasil

E-mail: pedrovitorp2@hotmail.com

Maria Isabelly Reis Teixeira

ORCID: https://orcid.org/0000-0002-8177-4379

Universidade Federal do Piauí, Brasil

E-mail: maria-belly@hotmail.com

Maria de Fátima Silva

ORCID: https://orcid.org/0000-0002-4422-6947

Centro Universitário de Ciências e Tecnologia do Maranhão, Brasil

E-mail: maryaf10@hotmail.com

Maria Gabriela de Sousa Bacelar

ORCID: https://orcid.org/0000-0002-5521-5258

Universidade Estadual do Maranhão, Brasil

E-mail: gabi.maria1@outlook.com 


\author{
Amanda Thais Franco Oliveira \\ ORCID: https://orcid.org/0000-0002-4210-7485 \\ Centro Universitário de Ciências e Tecnologia do Maranhão, Brasil \\ E-mail: amandafrancooliv@hotmail.com \\ Ana Luísa de Sousa Ferreira \\ ORCID: https://orcid.org/0000-0002-3398-6684 \\ Centro Universitário de Ciências e Tecnologia do Maranhão, Brasil \\ E-mail: analuisa2408991@ outlook.com.br \\ Edilene Ferreira da Costa Santos \\ ORCID: https://orcid.org/0000-0001-7304-9401 \\ Centro Universitário de Ciências e Tecnologia do Maranhão, Brasil \\ E-mail: dilla.soares@hotmail.com \\ Alessandra Ferreira Das Chagas \\ ORCID: https://orcid.org/0000-0002-2407-0666 \\ Centro Universitário de Ciências e Tecnologia do Maranhão, Brasil \\ E-mail: 18alenzo@gmail.com \\ Luana Pereira Ibiapina Coêlho \\ ORCID: https://orcid.org/0000-0002-2054-959X \\ Faculdade de Venda Nova do Imigrante, Brasil \\ E-mail: luana_ibiapina@hotmail.com \\ Pedro Henrique Medeiros de Andrade \\ ORCID: https://orcid.org/0000-0003-1819-9540 \\ Centro Universitário de Ciências e Tecnologia do Maranhão, Brasil \\ E-mail: pedro.medeiroscx@gmail.com \\ Francisca Tatiana Dourado Gonçalves \\ ORCID: https://orcid.org/0000-0001-5414-0381 \\ Universidade Federal do Pará, Brasil \\ E-mail: tatyanadourado@yahoo.com.br
}

\title{
Resumo
}

O presente trabalho teve como objetivo investigar a música e seus efeitos no controle da ansiedade em idosos que se encontravam em instituições de longa permanência. É uma pesquisa de abordagem quantitativa e qualitativa. Foram pesquisados 08 idosos atendidos em uma Instituição de Longa Permanência, localizada na cidade de Caxias Maranhão. Para o levantamento dos dados utilizou-se de técnicas de observação, entrevista semi-dirigida, questionário de caracterização sociodemográfica, diário de campo e aplicação da Escala de Ansiedade de Hamilton, instrumento utilizado para mensurar o grau de ansiedade dos entrevistados. Nos resultados alcançados verificou-se que cerca de $50 \%$ dos idosos apresentaram, na primeira avaliação sobre estado ansioso, um grau de ansiedade classificado como grau leve, moderado e grave e que após a aplicação das 05 sessões de repertório musicais, os mesmos apresentaram uma diminuição nesses graus, indo para uma classificação mínimo e moderado. Identificou-se uma menor prevalência dos graus de ansiedade nos idosos de sexo masculino. Verificou-se também que ao entrar em contato com a música os indivíduos resgataram algumas memórias, o que é de extrema importância para a população da terceira idade, pois mantém as funções cognitivas ativas. Deste modo, os resultados mostram indícios que a música pode ser utilizada como um instrumento terapêutico para promover um equilíbrio no estado ansioso da população idosa.

Palavras-chave: Idoso; Música; Instituição de longa permanência; Ansiedade.

\begin{abstract}
This study aimed to investigate music and its effects on anxiety control in elderly people who were in long-term care facilities. It is a research with a quantitative and qualitative approach. Eight elderly people cared for at a Long-Term Care Institution, located in the city of Caxias - Maranhão, were surveyed. For data collection, observation techniques, semi-directed interviews, sociodemographic characterization questionnaire, field diary and application of the Hamilton Anxiety Scale, an instrument used to measure the degree of anxiety of the interviewees, were used. In the results achieved, it was found that about $50 \%$ of the elderly presented, in the first assessment on an anxious state, a degree of anxiety classified as mild, moderate and severe and that after the application of the 05 musical repertoire sessions, they presented a decrease in these degrees, going to a minimum and moderate rating. A lower prevalence of degrees of anxiety was identified in elderly males. It was also found that upon contact with music, individuals recovered some memories, which is extremely important for the elderly population, as it keeps the cognitive functions active. In this way, the results show evidence that music can be used as a therapeutic tool to promote a balance in the anxious state of the elderly population.
\end{abstract}

Keywords: Aged; Music; Long-stay institutions; Anxiety.

\section{Resumen}

Este estudio tuvo como objetivo investigar la música y sus efectos sobre el control de la ansiedad en personas mayores que se encontraban en centros de atención a largo plazo. Es una investigación con enfoque cuantitativo y cualitativo. Se encuestó a ocho personas mayores atendidas en una Institución de Atención a Largo Plazo, ubicada en la ciudad de Caxias - Maranhão. Para la recolección de datos se utilizaron técnicas de observación, entrevistas semidirigidas, 
cuestionario de caracterización sociodemográfica, diario de campo y aplicación de la Escala de Ansiedad de Hamilton, instrumento utilizado para medir el grado de ansiedad de los entrevistados. En los resultados alcanzados, se encontró que alrededor del $50 \%$ de los ancianos presentaban, en la primera evaluación sobre un estado ansioso, un grado de ansiedad clasificado como leve, moderado y severo y que luego de la aplicación de las 05 sesiones de repertorio musical, presentó una disminución en estos grados, pasando a una calificación mínima y moderada. Se identificó una menor prevalencia de grados de ansiedad en varones ancianos. También se encontró que al contacto con la música, los individuos recuperan algunos recuerdos, lo cual es sumamente importante para la población anciana, ya que mantiene activas las funciones cognitivas. Así, los resultados muestran evidencia de que la música se puede utilizar como herramienta terapéutica para promover un equilibrio en el estado ansioso de la población anciana.

Palabras clave: Anciano; Música; Instituciones de larga estancia; Ansiedad.

\section{Introdução}

A música tem-se destacado em vários segmentos, no campo social e no campo da saúde, ela é principalmente, percebida pela audição, órgão do sentido, pertencente à divisão do sistema nervoso periférico. Mas, também, pode ser sentida pela reverberação das ondas sonoras, através das estruturas corporais. Desse modo, após ser captada, estimula o sistema nervoso autônomo (SNA), provocando efeitos, inicialmente, na esfera física, e posteriormente disseminada para os campos cognitivos, psicoemocionais, sociais e espirituais (Weinberger, 1999).

A Música como estratégia de promoção da saúde mental, promove impactos que podem ser positivos, quando associados ao bem-estar, recordações e melhora das relações sociais, ou negativos, de acordo com as preferências musicais ou mensagens contidas. A música colabora na constituição de vínculos e no desenvolvimento de mudanças pessoais e coletivas. Promovendo expressão de emoções e percepção, fortalecendo os recursos psicossociais e contribuindo para o bem estar e qualidade de vida. (Batista \& Ribeiro, 2016).

Sendo assim a música pode ser inserida em repertórios terapêuticos favorecendo a estabilidade do estado emocional dos indivíduos, já que ela provoca sensações ligadas a serotonina, o hormônio da alegria, instiga a recordação, imaginação e vários sentimentos pois ela produz efeito ansiolítico por estar vinculada a uma carga afetiva, e que, ao gerar prazer, diminui a ansiedade (Melo et al., 2017).

A ansiedade é um componente natural da vida, anunciando que algo perigoso pode ocorrer. No idoso a ansiedade está relacionada às limitações vivenciadas nessa etapa. Pessoas com alto nível de ansiedade questionam suas habilidades intelectuais e costumam antecipar sua inabilidade, prejudicando o raciocínio e a compreensão, o que nessa fase da vida poderia ser diferente com uma boa saúde psíquica (De Souza, 2017).

Em relação aos idosos institucionalizados, Machado et al. (2016) destacam que a institucionalização torna o idoso isolado da sociedade, causando diminuição da autoestima, perda da identidade e, consequentemente, fazendo com que se sinta desvalorizado. A fragmentação emocional é uma possibilidade presente quando a família entrega o idoso aos cuidados de instituições de longa permanência, abrindo possibilidades para o desenvolvimento de sentimentos de abandono, rejeição, tristeza e angustia. Esses sentimentos interferem na saúde emocional do sujeito interferindo e comprometendo o repertorio comportamental (Frade, Barbosa, Cardoso, \& Nunes, 2015).

Assim, ao se conectar musicalmente, o idoso institucionalizado pode expressar melhor seus sentimentos como medo, raiva, felicidade e alegria, possibilitando uma ressignificação desses sentimentos e da própria existência, podendo melhorar o contato com familiares, amigos e diminuindo a possibilidade de solidão.

A música por ser um recurso terapêutico aplicado no campo das ciências sociais, saúde e educação faz se necessária uma constância na fomentação de suas aplicabilidades e mensurar os resultados. Desse modo com o intuito de aprimorar o conhecimento da música e sua influência de intervenção no âmbito da saúde mental a pesquisa objetivou-se analisar a música como um instrumento terapêutico no controle da ansiedade nos idosos institucionalizados. 


\section{Metodologia}

Esta pesquisa teve como metodologia, estudo exploratório, descritivo de corte transversal, com abordagem qualitativa e quantitativa. $\mathrm{O}$ método quantitativo auxilia as interpretações de dados para um melhor embasamento, onde a abordagem qualitativa por ser definida como aquela que se preocupa com um nível de realidade que não pode ser quantificado e que trabalha com o universo dos significados, motivos, valores e atitudes, que, por sua vez, correspondem a um espaço mais profundo das relações, dos processos e dos fenômenos que não podem ser reduzidos à operacionalização de variáveis.

Participaram do estudo, idosos residentes em instituições de longa permanência localizados na cidade de Caxias Maranhão, a instituição é uma Associação Privada fundada em 16/12/2002. Sua atividade principal é o amparo ao idoso, atualmente a instituição atende 17 idosos. Foram aplicados como critério de inclusão idosos que estejam legalmente assistidos em instituições de longa permanência, que estejam com sua integridade psíquica preservada para assim poderem expressar a experiência vivenciada, podendo assim não interferir na fidedignidade dos resultados. Foram excluídos os idosos que apresentam diagnósticos médico de Transtornos Mentais. A amostra foi do tipo probabilística aleatória simples onde todos os sujeitos têm idêntica probabilidade de serem selecionados para a amostra. Contudo dos 18 idosos atendidos pela clínica de repouso de longa permanência, 08 fizeram parte da amostra do estudo. Foram excluídos 10 idosos pelos motivos: 02 com diagnostico de mal de Alzheimer, 03 deficientes auditivos, 02 com demência mista, 03 com esquizofrenia.

A coleta dos dados foi realizada na própria clínica de repouso entre os meses de abril e maio de 2019, aceitaram participar voluntariamente da pesquisa e declararam isso pela assinatura do termo de consentimento livre e esclarecido. Para obtenção de dados sociodemográficos e clínicos foi elaborado um questionário com perguntas fechadas, uma entrevista com perguntas abertas e aplicado à escala de Ansiedade de Hamilton, um instrumento traduzido e validado para o Brasil por Bertolucci, Brucki, Campacci e Juliano (1994). A escala é constituída por 14 itens, cada um definido por uma série de sintomas que mensura ansiedade psíquica e a ansiedade somática, uma vez que o objetivo do estudo foi usar a música como instrumento de controle da ansiedade na pessoa idosa.

O estudo foi aprovado pelo Comitê de Ética em Pesquisa do Centro Universitário de Ciências e Tecnologia do Maranhão-UNIFACEMA, sob o Protocolo n 3.221.356, conforme Resolução no 466/2012, do Conselho Nacional de Saúde.

Para a coleta dos dados, foram realizados 05 encontros, o primeiro encontro foi destinado para aplicação do questionário sociodemográfico, entrevista semi-dirigida, aplicação da escala de ansiedade e formulação do repertorio musical. O segundo, terceiro e quarto encontros foram direcionados para aplicação da música como instrumento terapêutico e o quinto e último encontro, serviu para reaplicação da escala de ansiedade de Hamilton. Todos os instrumentos foram aplicados de forma individualizada, sendo que o local e horário para aplicação dos instrumentos foram definidos de forma que ficassem convenientes aos participantes, sempre priorizando um clima de receptividade, segurança e confiabilidade.

Aplicação do questionário sociodemográfico serviu para elaboração do perfil de identificação da população da pesquisa, o inventário de ansiedade com o objetivo de evidenciar o nível de ansiedade em cada participante e entrevista semidirigida para a escolha dos repertórios musicais, ou seja, cada idoso apresentou de forma clara a sua preferência musical, no qual foram utilizados nas sessões posteriores, no último encontro foi aplicada a entrevista semidirigida com objetivo de verificar os efeitos da música no controle da ansiedade e dos sentimentos vivenciados durante a utilização do repertorio musical.

Após aplicação dos instrumentos realizou-se os procedimentos para análise dos dados, onde essa constituição foi dividida em três etapas: A primeira procedeu-se para o método quantitativo transcrevendo os dados para o programa Microsoft Excel. O segundo procedimento de análise foi a correção e mensuração do inventario de ansiedade Hamilton, procedendo todos os critérios de aplicação e correção, de acordo as instruções do manual do inventario utilizado. A avaliação qualitativa procedeu-se com a transcrição fidedigna das falas mencionadas durante a entrevista semidirigida, seguindo a formação da 
categorização temática com base na análise do conteúdo, conforme estipula Bardin (2011). Esse procedimento envolve pré-análise e organização do material após leitura exaustiva (leitura flutuante), com organização dos dados e análise e compreensão dos discursos dos participantes.

\section{Resultados e Discussão}

A apresentação dos resultados e discursões foi estruturado em três etapas: a primeira refere-se à caracterização dos sujeitos participantes da pesquisa, a segunda relaciona-se a mensuração do nível de ansiedade dos idosos institucionalizados, e a terceira refere-se à exposição dos depoimentos sobre os sentimentos vivenciados durante a utilização do repertório musical.

A Tabela 1 apresenta, em números, a caracterização dos participantes da pesquisa, com as variáveis sexo, idade, escolaridade, tempo de permanência na instituição, renda, estado civil e número de filhos.

Tabela 1. Caracterização sociodemográfica dos pacientes analisados. Caxias, MA, Brasil, 2019.

\begin{tabular}{|c|c|c|c|}
\hline VARIÁVEIS & RESULTADO & $\mathbf{N}^{\circ}$ & $\%$ \\
\hline \multirow{2}{*}{ SEXO } & Feminino & 5 & $63 \%$ \\
\hline & Masculino & 3 & $38 \%$ \\
\hline \multirow{3}{*}{ IDADE } & 60 anos & 2 & $25 \%$ \\
\hline & 70 anos & 3 & $38 \%$ \\
\hline & Acima de 80 anos & 3 & $38 \%$ \\
\hline \multirow{2}{*}{ ESCOLARIDADE } & Analfabetos & 6 & $75 \%$ \\
\hline & Ensino fundamental completo & 2 & $25 \%$ \\
\hline \multirow{2}{*}{ TEMPO DE PERMANENCIA NA INSTITUIÇÃO } & 2 anos & 7 & $88 \%$ \\
\hline & Abaixo de 2 anos & 1 & $13 \%$ \\
\hline RENDA & Salário mínimo & 8 & $100 \%$ \\
\hline \multirow{3}{*}{ ESTADO CIVIL } & Solteiros & 4 & $50 \%$ \\
\hline & Divorciados & 1 & $13 \%$ \\
\hline & Viúvos & 3 & $38 \%$ \\
\hline \multirow{4}{*}{$\mathbf{N}^{\circ}$ DE FILHOS } & Sem filhos & 4 & $50 \%$ \\
\hline & 2 Filhos & 2 & $25 \%$ \\
\hline & 3 Filhos & 1 & $13 \%$ \\
\hline & 4 Filhos & 1 & $13 \%$ \\
\hline
\end{tabular}

Fonte: Dados da pesquisa (2019).

A amostra foi constituída de oito idosos, destes cinco (63\%) eram do sexo feminino e três (38\%) do sexo masculino. No que diz respeito à idade, a faixa etária variou de 60 a 99 anos, onde dois (25\%) possuíam entre 60 a 70 anos, três (38\%) possuíam entre 70 a 80 anos e três (38\%) possuíam idade acima de 80 anos. Na questão da escolaridade seis (75\%) são analfabetos e dois $(25 \%)$ possuem o ensino fundamental.

Em relação ao tempo de permanência na instituição sete (88\%) possuem mais de dois anos, enquanto apenas um (13\%) possui abaixo de dois anos. A renda de todos (100\%) é um salário mínimo. No que se refere ao estado civil quatro (50\%) são solteiros, um (13\%) é divorciado e três (38\%) são viúvos. Sobre a quantidade de filhos quatro (50\%) não possuem filhos, dois possuem dois filhos (25\%), um (13\%) possui três filhos e um (13\%) possui 04 filhos.

Os dados acima mostram a predominância do sexo feminino em relação ao sexo masculino entre os idosos institucionalizados. Tshiswaka e Pinheiro (2020) diz que este predomínio, em geral, pode ser explicado pelo fato de a população mundial e nacional feminina ser maior do que a masculina, além disso, as mulheres experimentam uma maior 
probabilidade de ficarem viúvas mais cedo e possuir condições econômicas desfavoráveis, o que pode predispor à institucionalização.

Quanto às condições financeiras, uma das características marcantes dos idosos no Brasil é a baixa condição socioeconômica, onde a maioria tem como fonte de renda a aposentadoria. Nesse estudo os idosos tinham renda de um salário mínimo, Lini, Portella e Doring (2016) falam que um dos motivos que levam idosos a serem institucionalizados por seus familiares é a baixa condição financeira para prestar o cuidado em domicílio.

Constatou-se também que, metade dos idosos institucionalizados são solteiros e sem filhos, o que pode ser um fator influenciador para viverem institucionalizados. Mais de 50\% dos idosos não são alfabetizados. Godley et al. (2003) relaciona a falta de escolaridade na população idosa atual, ao fato de que nas décadas de 1930 até 1950, o Ensino Médio (antigo Escola Normal) era restrito a algumas classes sociais tidas como privilegiadas, atribui-se também o fato de que esses idosos viveram em uma época que o ensino não era tido como prioridade e por isso a não agregação ao sistema educacional.

Com a utilização da escala de Ansiedade de Hamilton - HAM-A foi possível verificar o nível de ansiedade dos participantes ao iniciarem a pesquisa, como demonstra a tabela abaixo:

Tabela 2. Nível de ansiedade dos entrevistados antes da utilização do repertório musical. Caxias, MA, Brasil, 2019.

\begin{tabular}{l|c|c|l}
\hline \multicolumn{1}{c|}{ Nível de ansiedade } & $\mathbf{N}^{\circ}$ & $\%$ & \multicolumn{1}{c}{ Sintomas } \\
\hline Mínimo & 02 & $25 \%$ & $\begin{array}{l}\text { Sensação de constipação, boca seca, mão molhada, aumento } \\
\text { de dores abdominais, taquicardia, ondas de frio, cefaleia e } \\
\text { sensação de fraqueza. }\end{array}$ \\
\hline Leve & 01 & $13 \%$ & $\begin{array}{l}\text { Sensações de fraqueza, dificuldade de concentração, } \\
\text { cefaleia, insônia, preocupações, sudorese. }\end{array}$ \\
\hline Moderado & 01 & $13 \%$ & $\begin{array}{l}\text { Tremores, dores torácicas, dificuldade de concentração, } \\
\text { sudorese, insônia, preocupações, medo de ficar sozinho, } \\
\text { sensação de opressão. }\end{array}$ \\
\hline Grave & 04 & $\begin{array}{l}\text { Dores musculares, rigidez muscular, medo de ficar sozinho, } \\
\text { sudorese, insônia, aumento da frequência urinária, dores } \\
\text { abdominais, taquicardia, ondas de frio. }\end{array}$ \\
\hline
\end{tabular}

Fonte: Pesquisa direta (2019).

O grupo estudado caracterizou-se por apresentar uma prevalência de sintomas ansiosos com escores de grave a mínimo, onde 50\% da população apresenta-se com nível de ansiedade grave, 13\% moderado e leve, e 25\% mínimo. Sendo que os sintomas de humor ansioso, cognitivo e ansiedade somática estão presentes nos indicadores dos sintomas manifestados, ou seja, estão relacionados a sintomas como sensação de constipação, cefaleia, dores musculares, espasmos musculares, tremores, sensação de sufocamento, dificuldade de concentração, insônia, preocupações, medo de ficar sozinho, sensação de opressão, sudorese, boca seca, mão molhada e aumento da frequência urinaria, dores abdominais, taquicardia, ondas de frio, visão turva e sensação de fraqueza.

Segundo Dalgalarrondo (2008), no caso de ansiedade, são frequentes sintomas como insônia, tensão, angústia, irritabilidade, depressão, dificuldade de concentração, bem como sintomas físicos como taquicardia, tontura, cefaléia, dores musculares, formigamento, suor. Byrne (2007) destaca que são frequentes sintomas de ansiedade em idosos, e na maioria das vezes, a ansiedade vem associada a transtornos depressivos e a doenças físicas e ainda provoca sintomas psíquicos e somáticos. Algumas dores físicas podem ser resposta do organismo a um quadro de ansiedade, muito comum em idosos institucionalizados. Xavier, Ferraz, Bertollucci, Poyares e Moriguchi (2001) estudaram a ansiedade em idosos com mais de 80 anos e constataram que 10,6\% dos 77 idosos da amostra apresentavam transtorno de ansiedade generalizada e elevados níveis de ansiedade, representados por sintomas somáticos e físicos. 
Para demonstrar os efeitos da música como recurso terapêutico, apresenta-se o gráfico com os dados após a aplicação de 04 sessões de músicas como instrumento terapêutico. Registrando-se o nível de ansiedade. Analisou-se os resultados da HAM-A e que se encontram consolidados e descritos da seguinte forma: quanto ao nível de ansiedade 50\% estão em classificação mínima e 25\% descrevem-se em ansiedade moderado e grave. As descrições dos sintomas permaneceram nas mesmas dimensões sintomáticas de humor ansioso, cognitivo e ansiedade (Figura 1).

Figura 1. Níveis de ansiedade antes e após aplicação dos repertórios musicais. Caxias, MA, Brasil, 2019.

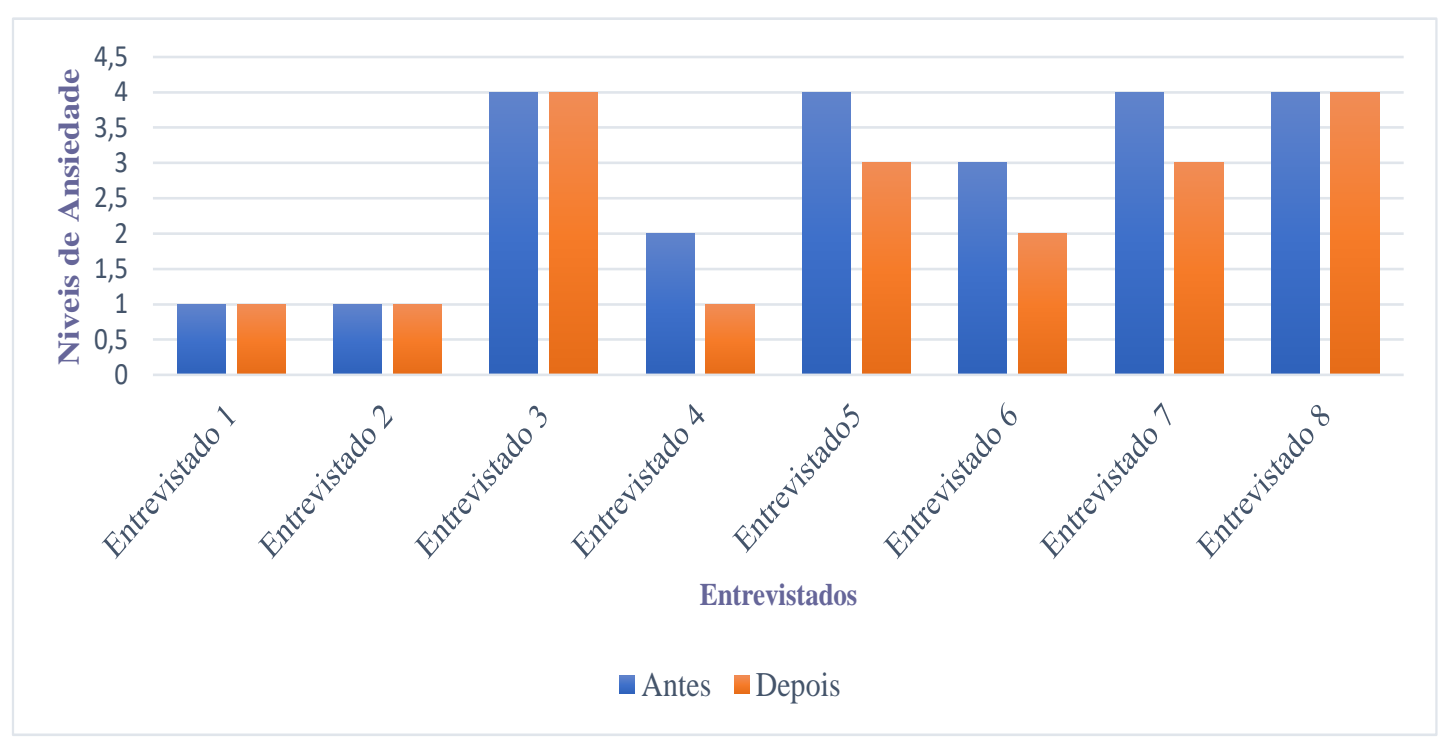

Legenda: Níveis de Ansiedade: Mínimo = 1; Leve=2; Moderado: 3; Grave=4. Fonte: Pesquisa direta (2019).

No gráfico podemos observar que a intensidade do quadro ansioso, segundo os resultados da HAM-A, diminuiu de maneira significativa, após a aplicação do repertório musical. Os dados apresentados mostram que os entrevistados 1, 2, 3 e 8 após a aplicação do repertório musical permaneceram com os mesmos níveis de ansiedade, não apresentando mudança com a aplicação da intervenção, continuando assim no mesmo grau de ansiedade que foi encontrado antes da intervenção. Já o entrevistado 4, possuía ansiedade grau leve, após a aplicação apresentou grau mínimo de ansiedade, o entrevistado 5 possuía ansiedade grau grave, e após a aplicação apresentou grau moderado, entrevistado 6 possuía ansiedade grau moderado, após aplicação apresentou grau mínimo e o entrevistado 7, possuía ansiedade grau grave e após a aplicação apresentou grau moderado. Isso mostra que na aplicação surgiu um efeito bastante significativo fazendo com que a ansiedade diminuísse com a aplicação do repertório musical.

Gonçalez, Nogueira e Puggina (2006) em uma pesquisa realizada com idosos, relata que a música afeta as emoções, atuando diretamente sobre os estados emocionais, proporcionando um melhor estado emocional e ajudando na redução do nível de ansiedade. Leão (2008) corrobora com os resultados acima ao descrever os benefícios que a música gera ao idoso, dentre eles são: relaxamento muscular, alívio do controle de ansiedade, melhora na participação e envolvimento nas atividades físicas, todos despertados através de aplicações musicais, onde os sujeitos obtiveram uma melhor qualidade de vida.

Por meio da entrevista semidirigida investigou-se os sentimentos vivenciados durante a utilização do repertorio musical, desdobrando-se metodologicamente a análise qualitativa desta pesquisa. A análise de conteúdo referente aos sentimentos vivenciados durante o repertorio musical gerou uma rubrica denominada memórias emocionais. O questionamento norteador explorou sobre os sentimentos vivenciados durante a aplicação da música como instrumento terapêutico. 
Os relatos apresentam uma descrição inicial de saudosismo, recordações de vivências do passado e melancolia. Como apresentam-se as narrativas descritas.

"Fiquei emocionada, pois essa música me traz saudades de minha filha".

"Essas músicas me fez lembrar meu marido, sinto muita saudade dele”.

"Me lembrei do meu único namorado que tive, por que ele queria casar e ir embora para o Rio de Janeiro, mas não tive coragem de deixar minha mãe"

A música proporciona o estabelecimento de um contato dos idosos com o resgate de memórias, caracterizando-se como elemento evocador de lembranças relacionadas à sua escuta, percebeu-se que a música se fez presente e acessou suas memórias afetivas de maneira que os mesmos reproduzissem lembranças vivencias no passado permitindo a descrição de relatos de trechos de suas vidas (Ziv, Granot, Hai, Dassa, \& Haimov 2007). Quanto aos sentimentos de tranquilidade, satisfação, bem-estar e prazer. Tais fatos podem ser observados nas falas abaixo:

"Quando ouço essas canções sinto uma paz muito grande em meu coração, fico feliz!".

"Uma música dessas alegra a alma da gente”.

"Eu gosto muito de dançar, quando escuto musica dá logo vontade de levantar e sair dançando".

Segundo Ziv et al. (2007), abordam que as atividades por meio da música ajudam ao idoso a recordar as emoções e sentimentos outrora adormecidos, favorecendo meios de expressão dos mesmos como de euforia, ânimo, alegria entre outros, diminuindo a ansiedade. Em seus estudos Gagnon, Peretz e Fülöp (2009), ressalta que esses sentimentos fazem com que as células boas se fortaleçam, promovendo uma satisfação de recordação de momentos bons do passado tornando bastante significativo para eles

\section{Conclusão}

Os resultados deste estudo demostram que a música é capaz de provocar mudanças emocionais em idosos e que pode ser utilizada como um instrumento terapêutico promovendo alterações significativas no estado ansioso dessa população.

Evidenciou-se na pesquisa que os sentimentos vivenciados pelos idosos durante a utilização da música como instrumento, contribuíram na diminuição dos níveis de ansiedade e provocaram a sensação de bem-estar emocional. O resgate das memórias emocionais, e euforia.

Conclui-se que a música é um instrumento eficaz, de fácil acesso e de grande relevância no controle dos sintomas ansiosos. Diante do exposto faz- se necessárias mais pesquisas aprofundadas, no que se refere à música como recurso de controle da ansiedade em idosos institucionalizados.

\section{Referências}

Bardin, L. (2011). Análise de conteúdo. Edições 70.

Batista, N. S., \& Ribeiro, C. M. (2016). O uso da música como recurso terapêutico em saúde mental. Revista De Terapia Ocupacional, 27(3), 336-341.

Bertolucci, P. H. F., Brucki, S. M. D., Campacci, S. R., \& Juliano, Y. (1994). O Mini exame do Estado Mental em uma população geral. Impacto da escolaridade. Arquivos de Neuropsiquiatria, 52(1), 1-7.

Byrne, R. M. J. (2007). Précis de The rational imagination: How people create alternatives to reality. Behavioral and Brain Sciences, 30, 439-480.

Dalgalarrondo, P. (2008). Psicopatologia e semiologia dos transtornos mentais. (2a ed.), Artmed. 
Research, Society and Development, v. 10, n. 7, e54610716780, 2021

(CC BY 4.0) | ISSN 2525-3409 | DOI: http://dx.doi.org/10.33448/rsd-v10i7.16780

De Souza, T. T. (2017). Laboratório online de música e tecnologia: planejando e implementando um MOOC para o ensino de Música online (Dissertação de Mestrado). Universidade de Brasília.

Frade, J., Barbosa, P., Cardoso, S., \& Nunes, C. (2015). Depressão no idoso: sintomas em indivíduos institucionalizados e não institucionalizados. Revista de Enfermagem Referência, (4), 41-9.

Gagnon, L., Peretz, I., \& Fülöp, T. (2009). Musical structural determinants of emotional judgments in dementia of the Alzheimer type. Neuropsychology, 23(1), 90-7.

Godley, P., Nguyen, A., Yokoyama, K., Rohack, J., Woodward, B., \& Chiang, T. (2003). Melhorando o cuidado com hipertensão em um grande grupo de modelo MCO. American Journal of Health-System Pharmacy, 60 (6), 554-564.

Gonçalez, D. F. C., Nogueira, A. T. O. Puggina, A. C. G. (2008). O uso da música na assistência de enfermagem no brasil: uma revisão bibliográfica. Cogitare Enfermagem, 13(4), 591-6.

Leão, E. R. (2008). A dignidade dos idosos institucionalizados: o papel da música no encontro humano. Revista eletrônica Enfermeria Global, (13), 1-6.

Lini, E. V., Portella, M. R., \& Doring, M. (2016). Fatores associados à institucionalização de idosos: estudo caso-controle. Revista Brasileira de Geriatria e Gerontologia, 19(6), 1004-1014.

Machado, M. B., Ignácio, Z. M., Jornada, L. K., Réus, G. Z., Abelaira, H. M., Arent, C. O., Schwalm, M. T., Ceretta, R. A., Ceretta, L. B., \& Quevedo, J. (2016). Prevalência de transtornos ansiosos e algumas comorbidades em idosos: um estudo de base populacional. Jornal Brasileiro de Psiquiatria, 65(1), 2835 .

Melo, G. A. A., Rodrigues, A. B., Firmeza, M. A., Grangeiro, A. S. M., Oliveira, P. P., \& Caetano, J. Á. (2017). Musical intervention on anxiety and vital parameters of chronic renal patients: a randomized clinical trial. Rev. Latino-Am. Enfermagem, 26, e2978.

Tshiswaka, S. K., \& Pinheiro, S. L. (2020) Avaliação do impacto da música como redutor de ansiedade no atendimento odontológico de crianças. Revista Gaúcha de Odontologia, 68, e20200033.

Weinberger, N. M. (1999). Music and the auditory system. In D. Deutsch (Ed.), Academic Press series in cognition and perception: A series of monographs and treatises. The psychology of music (p. 47-87). Academic Press.

Xavier F. M. F., Ferraz, M. P. T., Bertollucci, P., Poyares, D., \& Moriguchi, E. H. (2001). The prevalence of major depression and its impact in the quality of life, sleep patterns and cognitive function in a octogenarian population. Braz. J. Psychiatry 23 (2), 62-70.

Ziv, N., Granot, A., Hai, S., Dassa A., \& Haimov, I. (2007). The effect of background stimulative music on behavior in Alzheimer's patients. $J$ Music Ther., 44(4), 329-43. 